\title{
The calculation of a massive planar pentabox with a differential equation method
}

\author{
Costas G. Papadopoulos ${ }^{a}$, Damiano Tommasini ${ }^{a}$ and Christopher Wever ${ }^{\star a, b \dagger}$ \\ anstitute of Nuclear Physics, NCSR "Demokritos", Athens, Greece \\ ${ }^{b}$ Institute for Theoretical Particle Physics (TTP), Karlsruhe Institute of Technology, \\ Engesserstraße 7, D-76128 Karlsruhe, Germany \& Institute for Nuclear Physics (IKP), \\ Karlsruhe Institute of Technology, Hermann-von-Helmholtz-Platz 1, D-76344 \\ Eggenstein-Leopoldshafen, Germany \\ E-mail: costas.papadopoulos@cern.ch, tommasini@inp.demokritos.gr, \\ wevereinp.demokritos.gr
}

We present the calculation of massless two-loop Master Integrals relevant to five-point amplitudes with one off-shell external leg and derive the complete set of planar Master Integrals with five onmass-shell legs, that contribute to many $2 \rightarrow 3$ amplitudes of interest at the LHC, as for instance three jet production, $\gamma, V, H+2$ jets etc., based on the Simplified Differential Equations approach.

12th International Symposium on Radiative Corrections (Radcor 2015) and LoopFest XIV (Radiative Corrections for the LHC and Future Colliders)

15-19 June 2015

UCLA Department of Physics \& Astronomy Los Angeles, CA, USA

\footnotetext{
* Speaker.

${ }^{\dagger}$ Supported by the Research Funding Program ARISTEIA, HOCTools (co-financed by the European Union (European Social Fund ESF) and Greek national funds through the Operational Program "Education and Lifelong Learning" of the National Strategic Reference Framework (NSRF))
} 


\section{Introduction}

The LHC has started running at $13 \mathrm{TeV}$ in 2015 and in order to have a good comparison of the theoretical predictions with the new experimental results, a fully automated NNLO calculation framework is highly desirable. From the theoretical viewpoint, the reduction of one-loop amplitudes to a set of Master Integrals (MI) based on unitarity methods [1,2] and at the integrand level via the OPP method [3, 4], one-loop calculations have been fully automated in many numerical tools (some reviews are [5, 6]). Progress has also has been made towards the extension of these reduction methods to the two-loop order at the integral $[7,8,9]$ and the integrand $[10,11,12,13]$ level. Contrary to the MI at one-loop [14], a complete library of two-loops MI is missing.

A very fruitful method for calculating and expressing MI in terms of GPs is the differential equations (DE) approach [15, 16, 17, 18, 19], which has been used in the past two decades to calculate various MI at two-loops [20, 21, 22, 23, 24, 25]. In [26] a variant of the traditional DE approach to MI was presented, which was coined the Simplified Differential Equations (SDE) approach. In this talk we present an application of this method that first appeared in [27], concerning the calculation of planar massless MI relevant to five-point amplitudes with one off-shell leg, as well as the complete set of planar MI for five-point on-shell amplitudes. Pentabox integrals are needed in particular in order to compute NNLO QCD corrections to several processes of interest at LHC [28].

\section{The pentabox integrals}

The MI presented in this talk will be calculated with the SDE approach [26] and we refer to [29] for a detailed review ${ }^{1}$. We are interested in calculating the MI of two-loop QCD five-point amplitudes. As it is an inherent characteristic of the SDE method to interpolate among different kinematical configurations of the external momenta, the starting point is to compute five-point amplitudes with one off-shell leg. These amplitudes contribute to the production i.e., of one massive final state $V$, plus two massless final states $j_{1}, j_{2}$ at the LHC:

$$
p\left(q_{1}\right) p^{\prime}\left(q_{2}\right) \rightarrow V\left(q_{3}\right) j_{1}\left(q_{4}\right) j_{2}\left(q_{5}\right), q_{1}^{2}=q_{2}^{2}=0, q_{3}^{2}=M_{3}^{2}, q_{4}^{2}=q_{5}^{2}=0 .
$$

The colliding partons have massless momenta $q_{1}, q_{2}$, while the outgoing massive and the two massless particles have momenta $q_{3}$ and $q_{4}, q_{5}$ respectively. Of course, by appropriately taking the limit $M_{3}^{2} \rightarrow 0$ the pentabox MI with all external massless momenta on-shell will be obtained, that are relevant for instance to the three-jet production

$$
p\left(q_{1}\right) p^{\prime}\left(q_{2}\right) \rightarrow j_{1}\left(q_{3}\right) j_{2}\left(q_{4}\right) j_{3}\left(q_{5}\right), q_{i}^{2}=0 .
$$

For the off-shell case $M_{3}^{2} \neq 0$, there are in total three families of planar MI whose members with the maximum amount of denominators, namely eight, are graphically shown in Figure 1. Similarly, there are five non-planar families of MI as given in Figure 2. The two-loop planar (Fig. 1) and non-planar (Fig. 2) diagrams contributing to (2.1) have not been calculated yet. In this talk, all presented MI in the family $P_{1}$ as well as all the the on-shell ${ }^{2} \mathrm{MI}$ as $M_{3}^{2} \rightarrow 0$. We use the c++ implementation of the program FIRE [31] to perform the IBP reduction to the set of MI in $P_{1}$.

\footnotetext{
${ }^{1}$ See also the talk by C. Papadopoulos.

${ }^{2}$ Some results related to massless planar pentaboxes also appeared in [30].
} 

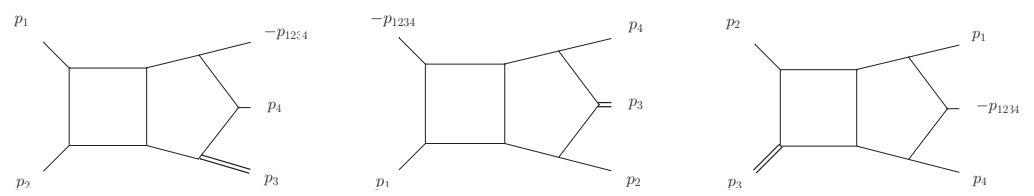

Figure 1: The three planar pentaboxes of the families $P_{1}$ (left), $P_{2}$ (middle) and $P_{3}$ (right) with one external massive leg.
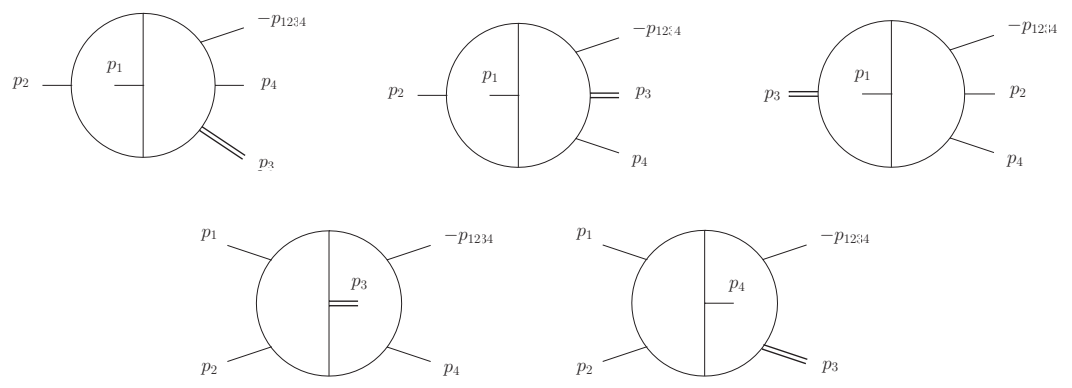

Figure 2: The five non-planar families with one external massive leg.
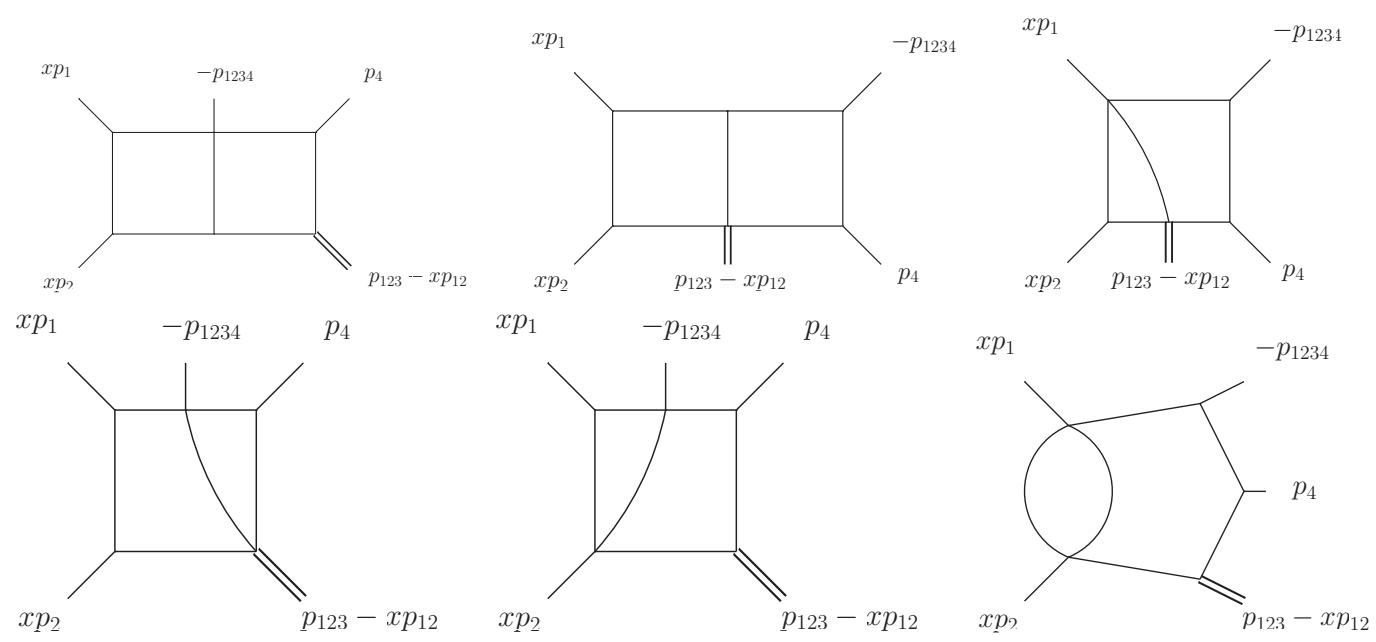

Figure 3: The five-point Feynman diagrams, besides the pentabox itself in Figure 1, that are contained in the family $P_{1}$. All external momenta are incoming.

The family $P_{1}$ contains in total 74 MI. There are seventeen new five-point Feynman diagrams that are not contained in the double box integral families [24, 25, 29]. Three of them are pentaboxes, including the scalar and two MI with irreducible numerators. There are six seven-denominator, and eight six-denominator ones, the scalar members of which are shown in Figure 3.

For the family of integrals $P_{1}$ the external momenta are parametrized in $x$ as shown in Figure 4. The MI in the family $P_{1}$ are therefore a function of a parameter $x$ and the following five invariants:

$$
s_{12}:=p_{12}^{2}, \quad s_{23}:=p_{23}^{2}, \quad s_{34}:=p_{34}^{2}, \quad s_{45}:=p_{45}^{2}=p_{123}^{2}, \quad s_{51}:=p_{15}^{2}=p_{234}^{2}, \quad p_{i}^{2}=0,
$$

where the notation $p_{i \cdots j}=p_{i}+\cdots+p_{j}$ is used and $p_{5}:=-p_{1234}$. As the parameter $x \rightarrow 1$, the 


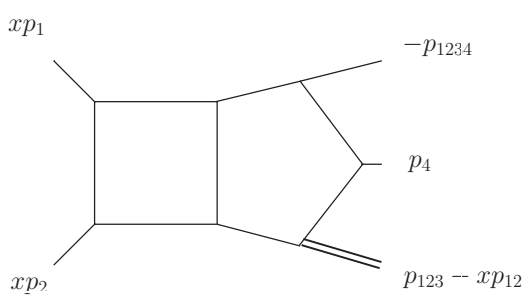

Figure 4: The parametrization of external momenta in terms of $x$ for the planar pentabox of the family $P_{1}$. All external momenta are incoming.

external momentum $q_{3}$ becomes massless, such that our parametrization (2.3) also captures the on-shell case $M_{3}^{2} \rightarrow 0$.

The MI depend in total on 6 variables, namely the Lorentz products $q_{i} \cdot q_{j}$ with $i<j<5$ and the (squared) particle mass $M_{3}^{2}=q_{3}^{2}$. The external momenta $q_{1}, q_{2}, q_{4}$ and $q_{5}$ of the massless external particles can correspond to either of the four massless external legs in Figure 4, while the massive particle $V$ has an external momenta $q_{3}=p_{123}-x p_{12}$ with a mass:

$$
M_{3}^{2}=(1-x)\left(s_{45}-s_{12} x\right) .
$$

After fixing the $x$-parameterization as in Figure 4, the class of loop integrals describing the planar family $P_{1}$ is now explicitly expressed in $x$ as:

$$
\begin{gathered}
G_{a_{1} \cdots a_{11}}^{P_{1}}(x, s, \varepsilon):=e^{2 \gamma_{E} \varepsilon} \int \frac{d^{d} k_{1}}{i \pi^{d / 2}} \frac{d^{d} k_{2}}{i \pi^{d / 2}} \frac{1}{k_{1}^{2 a_{1}}\left(k_{1}+x p_{1}\right)^{2 a_{2}}\left(k_{1}+x p_{12}\right)^{2 a_{3}}\left(k_{1}+p_{123}\right)^{2 a_{4}}} \\
\times \frac{1}{\left(k_{1}+p_{1234}\right)^{2 a_{5}} k_{2}^{2 a_{6}}\left(k_{2}-x p_{1}\right)^{2 a_{7}}\left(k_{2}-x p_{12}\right)^{2 a_{8}}\left(k_{2}-p_{123}\right)^{2 a_{9}}\left(k_{2}-p_{1234}\right)^{2 a_{10}}\left(k_{1}+k_{2}\right)^{2 a_{11}}},
\end{gathered}
$$

where $\gamma_{E}$ is the usual Euler-Mascheroni constant.

Using the notation given in Eq. (2.5), the indices $a_{1} \cdots a_{11}$ for the list of MI in the planar family $P_{1}$ is as follows ${ }^{3}$ :

$P_{1}:\{10000000101,01000000101,00100000101,10000001001,01000000011,00100000011,10100001100$, 10100001010, 10100101000,01000101001, 10100100100,10100000102,10100000101,10100000011, 10000001102, 10000001101, 10000001011,01000100101,01000001101,01000001011,00100100102, 00100100101,11100000101,11100000011,11000001102,11000001101,11000001012,11000001011, 11000000111, 10100000112, 10000001111,01100100102,01100100101,01100100011,01100000111, 01000101102,01000101101,01000101011,01000100111,01000001111,00100100111,10100101100, 10100100101, 10100001101,10100001011, 10100000111,111m0000111,110000m1111,11000001111, 10100101110, 10100100111,10100001111,011001m0111,01100100111,010m0101111,01000101111, 11100100101,11100001101,11100001011,11100000111,111m0101101,111001m1101,11100101101, 1110m101011,11100101011,111m0100111,11100100111,111000m1111,111m0001111,11100001111, $111001 \mathrm{~m} 0111,11100101111,111001 \mathrm{~m} 1111,111 \mathrm{~m} 0101111\}$,

In the next section we discuss the DE method that we use to calculate the above $74 \mathrm{MI}$ in $P_{1}$.

\footnotetext{
${ }^{3}$ The letter $\mathrm{m}$ is used here to indicate the index -1 .
} 


\section{Differential equations and their solution}

The resulting differential equation in matrix form can be written as

$$
\partial_{x} \mathbf{G}=\mathbf{M}\left(\left\{s_{i j}\right\}, \varepsilon, x\right) \mathbf{G}
$$

where $\mathbf{G}$ stands for the array of the $74 \mathrm{MI}$ given in Eq. (2.6). The diagonal part of the matrix at $\varepsilon=0$ defines as usual the integrating factors, $\left(M_{D}\right)_{I J}=\delta_{I J} M_{I I}(\varepsilon=0)$, and the equation takes the form $\partial_{x} \mathbf{G}=\mathbf{M G}$ with $\mathbf{G} \rightarrow \mathbf{S}^{-1} \mathbf{G}, \mathbf{S}=\exp \left(\int d x \mathbf{M}_{D}\right)$ and $\mathbf{M} \rightarrow \mathbf{S}^{-\mathbf{1}}\left(\mathbf{M}-\mathbf{M}_{D}\right) \mathbf{S}$.

We found [27] that, after absorbing the integrating factors, the resulting matrix $\mathbf{M}$ can be written as

$$
M_{I J}=N_{I J}(\varepsilon)\left(\sum_{i=1}^{20} \sum_{j=1}^{2} \sum_{k=0}^{1} \frac{C_{I J ; i j k} \varepsilon^{k}}{\left(x-l_{i}\right)^{j}}+\sum_{j=0}^{1} \sum_{k=0}^{1} \tilde{C}_{I J ; j k} \varepsilon^{k} x^{j}\right) .
$$

The twenty letters $l_{i}$, are given by

$$
\begin{aligned}
& 0,1, \frac{s_{45}}{s_{45}-s_{23}}, \frac{s_{45}}{s_{12}}, 1-\frac{s_{34}}{s_{12}}, 1+\frac{s_{23}}{s_{12}}, \\
& 1-\frac{s_{34}-s_{51}}{s_{12}}, \frac{s_{45}-s_{23}}{s_{12}},-\frac{s_{51}}{s_{12}}, \frac{s_{45}}{-s_{23}+s_{45}+s_{51}}, \frac{s_{45}}{s_{34}+s_{45}}, \\
& \frac{s_{12} s_{23}-2 s_{12} s_{45}-s_{12} s_{51}-s_{23} s_{34}+s_{34} s_{45}-s_{45} s_{51} \pm \sqrt{\Delta_{1}}}{2 s_{12}\left(s_{23}-s_{45}-s_{51}\right)}, \frac{s_{12} s_{23}-s_{12} s_{45}-s_{12} s_{51}-s_{23} s_{34}+s_{34} s_{45}-s_{45} s_{51} \pm \sqrt{\Delta_{2}}}{2 s_{12}\left(s_{23}-s_{45}-s_{51}\right)}, \\
& \frac{s_{12} s_{23}-s_{12} s_{51}-s_{23} s_{34}+s_{34} s_{45}-s_{45} s_{51} \pm \sqrt{\Delta_{1}}}{2 s_{12}\left(s_{23}+s_{34}-s_{51}\right)}, \quad \frac{s_{12} s_{45} \pm \sqrt{\Delta_{3}}}{s_{12} s_{34}+s_{12} s_{45}}, \quad \frac{s_{45}}{s_{12}+s_{23}},
\end{aligned}
$$

where

$$
\begin{aligned}
& \Delta_{1}=\left(s_{12}\left(s_{51}-s_{23}\right)+s_{23} s_{34}+s_{45}\left(s_{51}-s_{34}\right)\right)^{2}+4 s_{12} s_{45} s_{51}\left(s_{23}+s_{34}-s_{51}\right) \\
& \Delta_{2}=\left(s_{12}\left(-s_{23}+s_{45}+s_{51}\right)+s_{23} s_{34}+s_{45}\left(s_{51}-s_{34}\right)\right)^{2}-4 s_{12} s_{45} s_{51}\left(-s_{23}+s_{45}+s_{51}\right) \\
& \Delta_{3}=-\left(s_{12} s_{34} s_{45}\left(s_{12}-s_{34}-s_{45}\right)\right)
\end{aligned}
$$

with $\Delta_{1}$ being the usual Gram determinant. The normalization factors $N_{I J}(\varepsilon)$ can be cast in the factorized form $N_{I J}(\varepsilon)=n_{J}(\varepsilon) / n_{I}(\varepsilon)$ and can be absorbed by redefining $G_{I} \rightarrow n_{I}(\varepsilon) G_{I}$.

Although the DE can be solved from any form described so far, e.g. (3.1) and (3.2) and the result can be expressed as a sum of GPs with argument $x$ and weights given by the letters in Eq. (3.3), it is more elegant and easy-to-solve to derive a Fuchsian system of equations [32], where only single poles in the variable $x$ will appear. In fact the series of successive transformations

$$
\mathbf{G} \rightarrow\left(\mathbf{I}-\mathbf{K}_{i}\right) \mathbf{G}, \quad \mathbf{M} \rightarrow\left(\mathbf{M}-\partial_{x} \mathbf{K}_{i}-\mathbf{K}_{i} \mathbf{M}\right)\left(\mathbf{I}-\mathbf{K}_{i}\right)^{-1} i=1,2,3
$$

with

$$
\begin{gathered}
\left(\mathbf{K}_{1}\right)_{I J}=\left\{\begin{array}{cc}
\int d x(\mathbf{M}(\varepsilon=0))_{I J} & I, J \neq 69,72,73,74 \\
0 & I, J=69,72,73,74
\end{array}\right. \\
\left(\mathbf{K}_{2}\right)_{I J}=\left\{\begin{array}{cl}
\int d x(\mathbf{M}(\varepsilon=0))_{I J} & I, J \neq 74 \\
0 & I, J=74
\end{array}\right.
\end{gathered}
$$

and

$$
\left(\mathbf{K}_{3}\right)_{I J}=\int d x(\mathbf{M}(\varepsilon=0))_{I J}
$$


with the enumeration of the MI as given by Eq. (2.6), brings the system in to the form

$$
\partial_{x} \mathbf{G}=\left(\varepsilon \sum_{i=1}^{19} \frac{\mathbf{M}_{i}}{\left(x-l_{i}\right)}\right) \mathbf{G}
$$

where the residue matrices $\mathbf{M}_{i}$ are independent of $x$ and $\varepsilon$. It should be noticed that the series of the above transformations do not correspond to the one described by the Moser algorithm [33, 34, $35,36]$. The result can be straightforwardly given as

$$
\begin{aligned}
\mathbf{G} & =\varepsilon^{-2} \mathbf{b}_{0}^{(-2)} \\
& +\varepsilon^{-1}\left(\sum \mathscr{G}_{a} \mathbf{M}_{a} \mathbf{b}_{0}^{(-2)}+\mathbf{b}_{0}^{(-1)}\right) \\
& +\left(\sum \mathscr{S}_{a b} \mathbf{M}_{a} \mathbf{M}_{b} \mathbf{b}_{0}^{(-2)}+\sum \mathscr{S}_{a} \mathbf{M}_{a} \mathbf{b}_{0}^{(-1)}+\mathbf{b}_{0}^{(0)}\right) \\
& +\varepsilon\left(\sum \mathscr{S}_{a b c} \mathbf{M}_{a} \mathbf{M}_{b} \mathbf{M}_{c} b_{0}^{(-2)}+\sum \mathscr{S}_{a b} \mathbf{M}_{a} \mathbf{M}_{b} \mathbf{b}_{0}^{(-1)}+\sum \mathscr{S}_{a} \mathbf{M}_{a} \mathbf{b}_{0}^{(0)}+\mathbf{b}_{0}^{(1)}\right) \\
& +\varepsilon^{2}\left(\sum \mathscr{S}_{a b c d} \mathbf{M}_{a} \mathbf{M}_{b} \mathbf{M}_{c} \mathbf{M}_{d} \mathbf{b}_{0}^{(-2)}+\sum \mathscr{S}_{a b c} \mathbf{M}_{a} \mathbf{M}_{b} \mathbf{M}_{c} \mathbf{b}_{0}^{(-1)}+\sum \mathscr{S}_{a b} \mathbf{M}_{a} \mathbf{M}_{b} \mathbf{b}_{0}^{(0)}+\sum \mathscr{G}_{a} \mathbf{M}_{a} \mathbf{b}_{0}^{(1)}+\mathbf{b}_{0}^{(2)}\right)
\end{aligned}
$$

with the arrays $\mathbf{b}_{0}^{(k)}, k=-2, \ldots, 2$ representing the $x$-independent boundary terms in the limit $x=0$ at order $\varepsilon^{k}$. The expression is in terms of Goncharov polylogarithms, $\mathscr{G}_{a_{1} \cdots a_{n}}=\mathscr{G}\left(l_{a_{1}}, l_{a_{2}}, \ldots, l_{a_{n}} ; x\right)$.

The limit $x=1$ represents the solution for all planar pentabox on-shell Feynman integrals. The limit can easily obtained by properly resumming the $\log ^{k}(1-x)$ terms. Interestingly enough we found a very simple formula for this limit given by

$$
\mathbf{G}_{x=1}=\left(\mathbf{I}+\frac{3}{2} \mathbf{M}_{2}+\frac{1}{2} \mathbf{M}_{2}^{2}\right) \mathbf{G}_{\text {trunc }}
$$

with $\mathbf{M}_{2}$ the residue matrix at $x=1$ and $\mathbf{G}_{\text {trunc }}$ derived from Eq.(3.4), by properly removing all divergencies proportional to $\log ^{k}(1-x)$ and setting $x=1$.

For the majority of the MI in the original basis (2.6), their boundary behaviour are captured by the DE itself as was also the case for the doublebox families [29]. To explain this, we turn to the language of expansion by regions $[37,38]$ which states that all MI can be written as an expansion in terms of the form $a_{i j} x^{i+j \varepsilon}$. Since such functions are linearly independent, linear equations for the coefficients $a_{i j}$ can be found by plugging such expansions for the integrals in the DE. The boundary conditions correspond to the leading terms in the expansion as $x \rightarrow 0$ and thus they are described by the expansion terms $a_{i j} x^{i+j \varepsilon}$, where $i=i_{0}$ is the smallest integer such that $a_{i j}$ is nonvanishing. In this way the DE themselves set constraints on the coefficients $a_{i j}$ and therefore the boundary conditions.

In general a MI $G$ behaves at the boundary $x \rightarrow 0$ as follows [37, 38]:

$$
G_{r e s}=\lim _{x \rightarrow 0} G=\sum_{j} c_{j} x^{i_{0}+j \varepsilon}+d_{j} x^{i_{0}+1+j \varepsilon}+\mathscr{O}\left(x^{i_{0}+2}\right),
$$

where in the expression $G_{\text {res }}$, the logarithms $\log ^{k}(x)$ have been resummed into terms of the form $\sim x^{\alpha \varepsilon}$ at the boundary $x=0$. As explained above, by putting the above form (3.6) for the integrals in the DE and equating the terms $x^{i+j \varepsilon}$ with the same exponents on both sides of the DE, linear equations are found for the coefficients $c_{i}$ and $d_{i}$. We solved these linear equations for the coefficients in the original basis (2.6) from the bottom-up. In other words we first solved the linear equations for the non-trivial MI with least amount of denominators and then recursively solved the 
coefficients for those MI with more denominators. At every step we used the solutions for the coefficients of the MI with less denominators that were found in the previous steps. The expressions for the trivial MI, i.e. those that satisfy a homogeneous DE, are plugged in directly and contribute to the inhomogeneous part of the linear equations for the coefficients. We note here that for the large majority of integrals we did not need to solve for the coefficients $d_{i}$ that correspond to $x$-suppressed terms. Their calculations were only required for those integrals whose DE had singularities of the form $x^{-2+\alpha \varepsilon}$ at the boundary $x=0$ (such singularities were also encountered for the DE of the one-loop pentagon discussed in [26]). Once the resummed terms in equation (3.6) were calculated, the resulting DE for $G_{f i n}:=G-G_{\text {res }}$ have no singularities at $x=0$ and can be directly integrated to Goncharov polylogarithms [39, 40, 41].

For the pentabox family $P_{1}$ specifically, the majority of the coefficients are fixed by these equations, while some others are not. We found in practice that for most of the integrals, the coefficients which are not fixed by the linear equations are zero and we confirmed this by the method of expansion by regions. However, for some integrals we found that the method of expansion by regions predicts that some coefficients that are not determined by the linear equations, are in fact non-zero and require an explicit calculation. For those integrals we used various other methods to calculate the unknown and nonvanishing coefficients (cf. [27] for further details). Once all boundary conditions were found for the integrals in the original basis (2.6), the boundary conditions for the canonical basis followed directly from the relation between the two bases that is described above.

The complete expressions for all MI are available in the ancillary files [42]. The solution for all 74 MI contains $O(3,000)$ GPs which is approximately six times more than the corresponding double-box with two off-shell legs planar MI. We have performed several numerical checks of all our calculations. The numerical results, also included in the ancillary files [42], have been performed with the GiNaC library [43] and compared with those provided by the numerical code SecDec $[44,45,46,47,48]$ in the Euclidean region for all MI and in the physical region whenever possible (due to CPU time limitations in using SecDec) and found perfect agreement. For the physical region we used the analytic continuation as described in [29]. At the present stage we are not setting a fully-fledged numerical implementation, which will be done when all families will be computed. By using HyperInt [49] to bring all GPs in their range of convergence ${ }^{4}$, before evaluating them numerically by GiNaC, the complete library of $74 \mathrm{MI}$ contains $O(6,000)$ GPs that all admit a fast numerical evaluation by their series expansions. Moreover expressing GPs in terms of classical polylogarithms and $L i_{2,2}$, could also reduce substantially the CPU time [50, 51]. Based on the above we estimate that a target of $O\left(10^{2}-10^{3}\right)$ milliseconds can be achieved.

\section{Conclusions}

In this talk we presented results of one of the topologies of planar Master Integrals related to massless five-point amplitudes with one off-shell leg as well as the full set of massless planar Master Integrals for on-shell kinematics. It follows that based on the Simplified Differential Equations approach [26] these MI can be expressed in terms of Goncharov polylogarithms. The complexity of the resulting expressions is certainly promising that the project of computing all MI relevant

\footnotetext{
${ }^{4}$ See also the talk by D. Tommasini.
} 
to massless QCD, namely all eight-denominator MI with arbitrary configuration of the external momenta, is feasible. Having such a complete library of two-loop MI, the analog of $A_{0}, B_{0}, C_{0}, D_{0}$ scalar integrals at one loop, the reduction of an arbitrary two-loop amplitude à la OPP can pave the road for a NNLO automation in the near future.

\section{References}

[1] Z. Bern, L. J. Dixon, D. C. Dunbar, and D. A. Kosower, Fusing gauge theory tree amplitudes into loop amplitudes, Nucl.Phys. B435 (1995) 59-101, [hep-ph/9409265].

[2] Z. Bern, L. J. Dixon, D. C. Dunbar, and D. A. Kosower, One loop n point gauge theory amplitudes, unitarity and collinear limits, Nucl.Phys. B425 (1994) 217-260, [hep-ph/9403226].

[3] G. Ossola, C. G. Papadopoulos, and R. Pittau, Reducing full one-loop amplitudes to scalar integrals at the integrand level, Nucl.Phys. B763 (2007) 147-169, [hep-ph / 0609007 ].

[4] G. Ossola, C. G. Papadopoulos, and R. Pittau, On the Rational Terms of the one-loop amplitudes, JHEP 0805 (2008) 004, [arXiv: 0802 . 1876].

[5] SM AND NLO MULTILEG and SM MC Working Groups Collaboration, J. Alcaraz Maestre et al., The SM and NLO Multileg and SM MC Working Groups: Summary Report, arXiv: 1203.6803 .

[6] R. K. Ellis, Z. Kunszt, K. Melnikov, and G. Zanderighi, One-loop calculations in quantum field theory: from Feynman diagrams to unitarity cuts, Phys.Rept. 518 (2012) 141-250, [arXiv:1105.4319].

[7] J. Gluza, K. Kajda, and D. A. Kosower, Towards a Basis for Planar Two-Loop Integrals, Phys.Rev. D83 (2011) 045012, [arXiv: 1009 . 0472].

[8] D. A. Kosower and K. J. Larsen, Maximal Unitarity at Two Loops, Phys.Rev. D85 (2012) 045017 , [arXiv:1108.1180].

[9] S. Caron-Huot and K. J. Larsen, Uniqueness of two-loop master contours, JHEP 1210 (2012) 026, [arXiv:1205.0801].

[10] P. Mastrolia and G. Ossola, On the Integrand-Reduction Method for Two-Loop Scattering Amplitudes, JHEP 1111 (2011) 014, [arXiv:1107.6041].

[11] S. Badger, H. Frellesvig, and Y. Zhang, Hepta-Cuts of Two-Loop Scattering Amplitudes, JHEP 1204 (2012) 055, [arXiv:1202.2019].

[12] S. Badger, H. Frellesvig, and Y. Zhang, A Two-Loop Five-Gluon Helicity Amplitude in QCD, JHEP 1312 (2013) 045, [arXiv:1310.1051].

[13] C. Papadopoulos, R. Kleiss, and I. Malamos, Reduction at the integrand level beyond NLO, PoS Corfu2012 (2013) 019.

[14] G. 't Hooft and M. Veltman, Scalar One Loop Integrals, Nucl.Phys. B153 (1979) 365-401.

[15] A. Kotikov, Differential equations method: New technique for massive Feynman diagrams calculation, Phys.Lett. B254 (1991) 158-164.

[16] A. Kotikov, Differential equation method: The Calculation of N point Feynman diagrams, Phys.Lett. B267 (1991) 123-127. 
[17] Z. Bern, L. J. Dixon, and D. A. Kosower, Dimensionally regulated one loop integrals, Phys.Lett. B302 (1993) 299-308, [hep-ph/9212308].

[18] E. Remiddi, Differential equations for Feynman graph amplitudes, Nuovo Cim. A110 (1997) 1435-1452, [hep-th/9711188].

[19] J. M. Henn, Multiloop integrals in dimensional regularization made simple, Phys.Rev.Lett. 110 (2013), no. 25 251601, [arXiv:1304.1806].

[20] M. Caffo, H. Czyz, S. Laporta, and E. Remiddi, The Master differential equations for the two loop sunrise selfmass amplitudes, Nuovo Cim. A111 (1998) 365-389, [hep-th/9805118].

[21] T. Gehrmann and E. Remiddi, Differential equations for two loop four point functions, Nucl.Phys. B580 (2000) 485-518, [hep-ph/9912329].

[22] T. Gehrmann and E. Remiddi, Two loop master integrals for gamma* $\rightarrow 3$ jets: The Planar topologies, Nucl.Phys. B601 (2001) 248-286, [hep-ph/ 0008287$].$

[23] T. Gehrmann and E. Remiddi, Two loop master integrals for gamma* $\rightarrow 3$ jets: The Nonplanar topologies, Nucl.Phys. B601 (2001) 287-317, [hep-ph/ 0101124$].$

[24] J. M. Henn, K. Melnikov, and V. A. Smirnov, Two-loop planar master integrals for the production of off-shell vector bosons in hadron collisions, JHEP 1405 (2014) 090, [arXiv: 1402 . 7078 ].

[25] F. Caola, J. M. Henn, K. Melnikov, and V. A. Smirnov, Non-planar master integrals for the production of two off-shell vector bosons in collisions of massless partons, a XXiv: 1404.5590.

[26] C. G. Papadopoulos, Simplified differential equations approach for Master Integrals, JHEP 1407 (2014) 088, [arXiv:1401.6057].

[27] C. G. Papadopoulos, D. Tommasini, and C. Wever, The Pentabox Master Integrals with the Simplified Differential Equations approach, arXiv:1511.0940. arXiv:1511.09404.

[28] J. R. Andersen et al., Les Houches 2013: Physics at TeV Colliders: Standard Model Working Group Report, arXiv:1405.1067.

[29] C. G. Papadopoulos, D. Tommasini, and C. Wever, Two-loop Master Integrals with the Simplified Differential Equations approach, JHEP 01 (2015) 072, [arXiv: 1409.6114$].$

[30] T. Gehrmann, J. M. Henn, and N. A. L. Presti, Analytic form of the two-loop planar five-gluon all-plus-helicity amplitude in QCD, arXiv:1511.0540.

[31] A. V. Smirnov, FIRE5: a C++ implementation of Feynman Integral REduction, Comput. Phys. Commun. 189 (2014) 182-191, [arXiv:1408.2372].

[32] J. M. Henn, Lectures on differential equations for Feynman integrals, J. Phys. A48 (2015) 153001, [arXiv:1412.2296].

[33] R. N. Lee, Reducing differential equations for multiloop master integrals, JHEP 04 (2015) 108, [arXiv:1411.0911].

[34] M. Barkatou and E.Pflügel, On the Moser-and super-reduction algorithms of systems of linear differential equations and their complexity, Journal of Symbolic Computation 44 (2009) 1017-1036.

[35] M. Barkatou and E.Pflügel, Computing super-irreducible forms of systems of linear differential equations via Moser-reduction: a new approach, Proceedings of the 2007 international symposium on Symbolic and algebraic computation ACM (2007) 1-8.

[36] J.Moser, The order of a singularity in Fuchs' theory, Mathematische Zeitschrift 72 (1960) 379-398. 
[37] M. Beneke and V. A. Smirnov, Asymptotic expansion of Feynman integrals near threshold, Nucl.Phys. B522 (1998) 321-344, [hep-ph/9711391].

[38] V. A. Smirnov, Applied asymptotic expansions in momenta and masses, Springer Tracts Mod.Phys. 177 (2002) 1-262.

[39] A. B. Goncharov, Multiple polylogarithms, cyclotomy and modular complexes, Math.Res.Lett. 5 (1998) 497-516, [arXiv:1105.2076].

[40] E. Remiddi and J. Vermaseren, Harmonic polylogarithms, Int.J.Mod.Phys. A15 (2000) 725-754, [hep-ph/9905237].

[41] A. Goncharov, Multiple polylogarithms and mixed Tate motives, math/ 0103059.

[42] available at https://www.dropbox.com/s/90iiqfcazrhwtso/results.tgz?dl=0.

[43] J. Vollinga and S. Weinzierl, Numerical evaluation of multiple polylogarithms, Comput.Phys.Commun. 167 (2005) 177, [hep-ph/ 0410259 ].

[44] T. Binoth and G. Heinrich, An automatized algorithm to compute infrared divergent multiloop integrals, Nucl.Phys. B585 (2000) 741-759, [hep-ph/ 0004013$].$

[45] G. Heinrich, Sector Decomposition, Int.J.Mod.Phys. A23 (2008) 1457-1486, [arXiv: 0803 . 4177].

[46] S. Borowka, J. Carter, and G. Heinrich, Numerical Evaluation of Multi-Loop Integrals for Arbitrary Kinematics with SecDec 2.0, Comput.Phys.Commun. 184 (2013) 396-408, [arXiv : 1204.4152 ].

[47] S. Borowka and G. Heinrich, Massive non-planar two-loop four-point integrals with SecDec 2.1, Comput.Phys.Commun. 184 (2013) 2552-2561, [arXiv: 1303.1157 ].

[48] S. Borowka, G. Heinrich, S. P. Jones, M. Kerner, J. Schlenk, and T. Zirke, SecDec-3.0: numerical evaluation of multi-scale integrals beyond one loop, Comput. Phys. Commun. 196 (2015) 470-491, [arXiv:1502.0659].

[49] E. Panzer, Algorithms for the symbolic integration of hyperlogarithms with applications to Feynman integrals, Comput. Phys. Commun. 188 (2014) 148-166, [arXiv: 1403.3385$].$

[50] T. Gehrmann, A. von Manteuffel, and L. Tancredi, The two-loop helicity amplitudes for $q \bar{q}^{\prime} \rightarrow V_{1} V_{2} \rightarrow 4$ leptons, JHEP 09 (2015) 128, [arXiv: 1503.0481 ].

[51] H. Frellesvig, D. Tommasini, and C. Wever, On the reduction of generalized polylogarithms to $\mathrm{Li}_{n}$ and $\mathrm{Li}_{2,2}$ and on the evaluation thereof, arXiv: 1601.0264. 